\title{
A Case of Rapid Eye Movement Sleep Behavior Disorder during Continuous Positive Airway Pressure Titration in a Patient with Multiple System Atrophy
}

\author{
Jung-Hwan Oh, Sook-Keun Song, Ji-Hoon Kang \\ Department of Neurology, Jeju National University School of Medicine, Jeju, Korea
}

Received November 8, 2017

Revised December 10, 2017

Accepted December 18, 2017

Address for correspondence

Ji-Hoon Kang, MD, $\mathrm{PhD}$

Department of Neurology,

Jeju National University Hospital,

15 Aran 13-gil, Jeju 63241, Korea

Tel: $+82-64-717-1620$

Fax: +82-64-717-1320

E-mail: jhkang@jejunu.ac.kr

\begin{abstract}
Sleep disorders are commonly observed in multiple systemic atrophy (MSA). The rapid eye movement (REM) sleep behavior disorder (RBD) is characterized by loss of normal voluntary muscle atonia during REM sleep. It usually presents during early course, and disappears over the course of disease progression. Sleep-disordered breathing (SDB) is also common sleep disorder in MSA which can be life-threatening, and continuous positive airway pressure (CPAP) treatment is useful in these patients. A 74-year-old woman with MSA presented for nocturnal respiratory disturbance. She had a five-year history of dream enacting behaviors, which had disappeared four months prior. Polysomnography revealed frequent stridor and sleep hypopnea. During the following full nigh CPAP titration for SDB, dream enacting behavior was observed during REM sleep stage. In MSA patients with SDB, CPAP administration may lead to increase REM sleep stage. An increase in REM sleep stage, which previously had been deprived, may have trigger RBD symptoms to reappear. The CPAP treatment should be considered with great caution in these patients.
\end{abstract}

J Sleep Med 2017;14(2):77-80

Key Words: Multiple system atrophy, REM sleep behavior disorder, Continuous positive airway pressure.
In patients with multiple system atrophy (MSA), sleep disorders, such as excessive daytime sleepiness, rapid eye movement (REM) sleep behavior disorder (RBD), and sleep-disordered breathing (SDB), are a common complaint. ${ }^{1}$ SDB can manifest as stridor, obstructive sleep apnea (OSA), and central sleep apnea in these patients. ${ }^{1}$ Central sleep apnea is more commonly reported in the later stages of MSA, ${ }^{1}$ and stridor is typically found together with OSA. ${ }^{2}$ For patients with MSA, SDB can be life-threatening, and continuous positive airway pressure (CPAP) is recommended as the first-line therapy. ${ }^{2}$ RBD develops in $90 \%$ to $100 \%$ of the patients, indicating a severe and extensive pathologic deficit in the central nervous system, which regulates REM sleep. ${ }^{2}$ In patents with MSA, RBD usually occurs just prior to or at the onset of MSA and then disappears over the course of disease progression. ${ }^{2}$ To our best knowledge, this is the first report of a MSA patient with SDB who developed RBD during CPAP titration.

This is an Open Access article distributed under the terms of the Creative Commons Attribution Non-Commercial License (http://creativecommons.org/licenses/by-nc/4.0) which permits unrestricted non-commercial use, distribution, and reproduction in any medium, provided the original work is properly cited.

\section{Case Report}

A 74-year-old woman presented to our hospital for evaluation of nocturnal respiratory disturbance and abnormal breathing sounds during sleep, which developed seven months prior to her initial visit. Four years earlier, she had noticed generalized bradykinesia with orthostatic hypotension and urge incontinence, and had been diagnosed with MSA with predominant parkinsonism. Her symptoms had gradually deteriorated, and she was bedridden at the time of presentation. Her husband reported that she had repeated nocturnal episodes of complex motor behaviors and sleep talking for 5 years, which had become less frequent and less severe over several months, and had disappeared four months prior to presentation. She had been administered 1,000 mg levodopa, $0.75 \mathrm{mg}$ pramipexole, $150 \mathrm{mg}$ itopride, $0.5 \mathrm{mg}$ clonazepam, and $5 \mathrm{mg}$ midodrine daily.

Overnight polysomnography (PSG) revealed frequent, severe, harsh high-pitched sounds in the inspiration phase (stridor) with many episodes of obstructive hypopnea (Fig. 1). The number of stridor events was $834.0 / \mathrm{h}$ of sleep time, and 


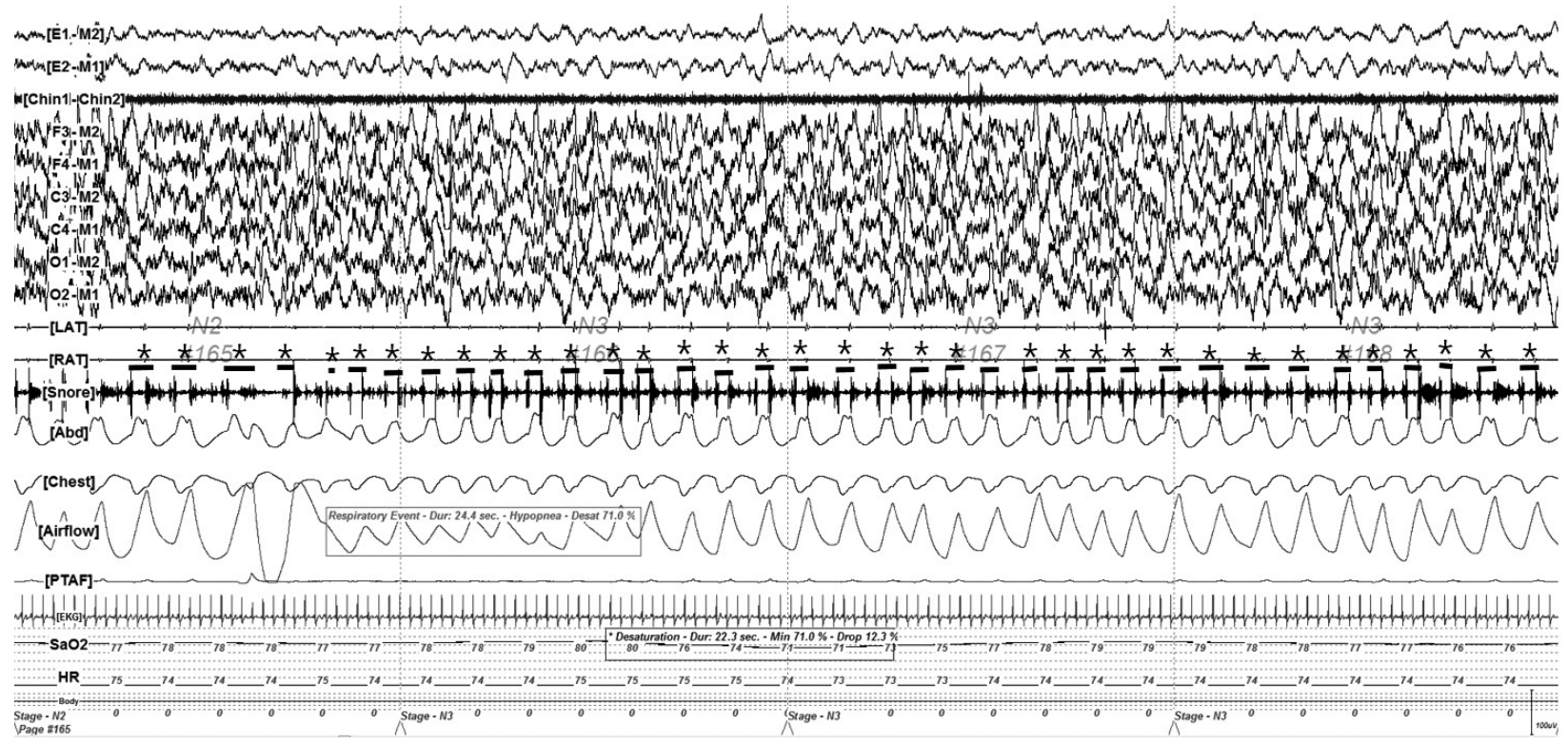

Figure 1. This 120 -s epoch polysomnogram demonstrates consistent periodic stridor ${ }^{*}$ ) reflected in a snore channel during slow wave sleep. It is a high-pitched, harsh sound during the inspiration phase. In addition, there are continuous episodes of oxygen desaturation, and an event of oronasal airflow limitations with a significant reduction of oxygen saturation corresponding to hypopnea. Top 2 channels: electrooculogram, Chin 1-Chin 2: chin electromyogram, six channels consisting of F3-M2, F4-M1, C3-M2, C4-M1, O1-M2, O2-M1: electroencephalogram, LAT: electromyogram on left anterior tibialis muscle, RAT: electromyogram on right anterior tibialis muscle, Snore: snore sensor, Abd: abdomen respiratory effort channel, Chest: chest respiratory effort channel, Airflow: oro-nasal thermal sensor, PTAF: pressure transducer air-flow, EKG: electrocardiography, SaO2: oxyhemoglobin saturation by pulse oximetry, HR: heart rate.

the apnea/hypopnea index (AHI) was $47.3 / \mathrm{h}$. The lowest oxyhemoglobin saturation $\left(\mathrm{SpO}_{2}\right)$ was $68 \%$, and low $\mathrm{SpO}_{2}$ was continuously measured (average $\mathrm{SpO}_{2}, 72.4 \%$ ). Sleep architecture showed an increase in stages $\mathrm{N} 1$ and $\mathrm{N} 2$ sleep, decreased stage N3 sleep, and absent REM sleep.

Six weeks after the initial PSG, CPAP titration with PSG was performed. CPAP was administered using 4 to $13 \mathrm{~cm} \mathrm{H}_{2} \mathrm{O}$. During the titration with $11 \mathrm{~cm} \mathrm{H}_{2} \mathrm{O}$ pressure, the patient had three episodes of talking and complex motor behaviors that were not observed in the initial PSG, with increased muscle tone during REM sleep, which was compatible with RBD (Fig. 2). REM sleep (3.7\% of total sleep stage) occurred after 244 minutes of latency, and was only present during the titration with $11 \mathrm{~cm} \mathrm{H}_{2} \mathrm{O}$ pressure. A fixed CPAP pressure of $11 \mathrm{~cm} \mathrm{H}_{2} \mathrm{O}$ was considered adequate for treatment of the SDB. With a pressure of $11 \mathrm{~cm} \mathrm{H}_{2} \mathrm{O}$, the $\mathrm{AHI}$ decreased to $4.7 / \mathrm{h}$, lowest $\mathrm{SpO}_{2}$ was $83 \%$, average $\mathrm{SpO}_{2}$ was $91.8 \%$, and stridor was nearly eliminated.

\section{Discussion}

Marked sleep fragmentation in patients with MSA has been reported by several polysomnographic studies and is related to motor symptoms such as urinary incontinence, rigidity, and bradykinesia with subsequent inability to turn over in the bed. ${ }^{2}$
Recently, two cases of RBD during CPAP titration were reported in Korean patients with OSA who developed RBD symptoms with increased REM sleep. ${ }^{3}$ OSA can also lead to sleep fragmentation; hence, patients with the disease show an increase in light non-REM sleep, and a decrease in REM sleep and slow-wave sleep on PSG. ${ }^{4}$ When CPAP is administered at the appropriate pressure level for OSA, respiratory obstructive events are eliminated; hence, the amounts of REM and slow-wave sleep increase. ${ }^{5,6}$

Extensive research has shown that SDB is a frequent and major problem in patients with MSA. ${ }^{1}$ One of the most typical types of SDB in MSA is stridor caused by upper airway obstruction that frequently accompanies OSA. ${ }^{2}$ Such SDB can cause sleep fragmentation in patients with MSA, and CPAP is recommended as a first-line treatment for increased long-term survival. ${ }^{7}$ In the present case, during a therapeutic trial of CPAP, when an adequate pressure level for the treatment of SDB was reached, REM sleep occurred, and consequently, RBD developed.

Although RBD is a common manifestation in patients with MSA, it does not present in the later stages of disease, and it can disappear with worsening disease condition. ${ }^{2,8}$ These phenomena can be explained by progressive degeneration of the brain stem nuclei, which control REM sleep. ${ }^{2,9}$ Furthermore, in cases like the present, sleep fragmentation might mask RBD in MSA patients with SDB. In addition, clonaze- 
A

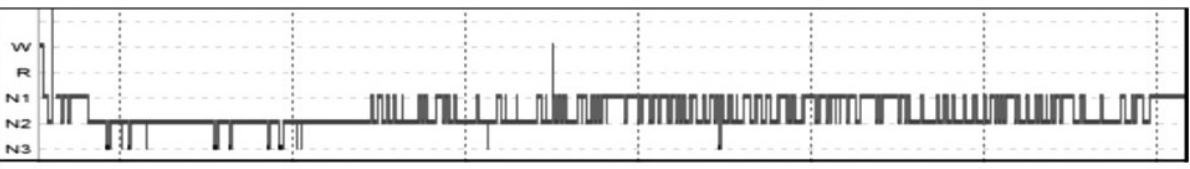

B
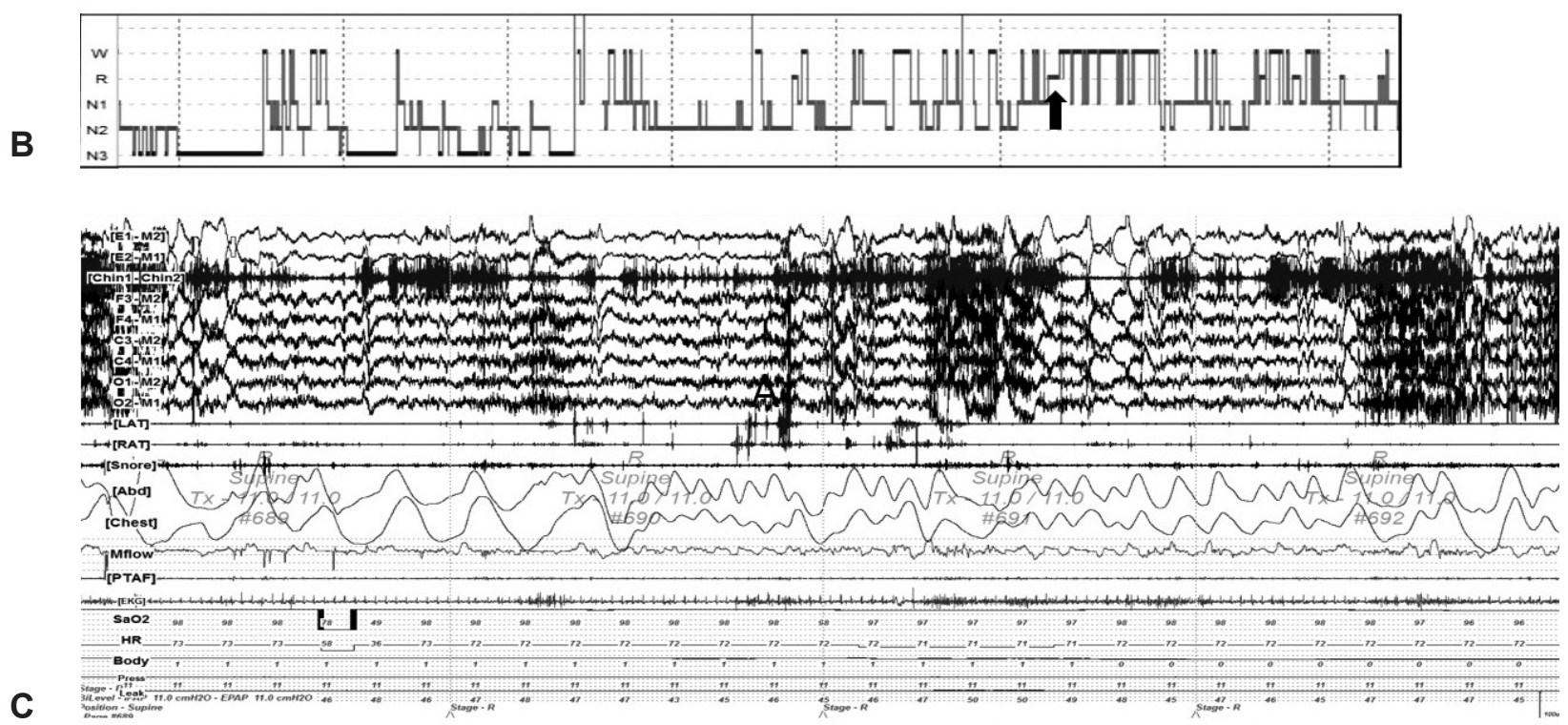

C
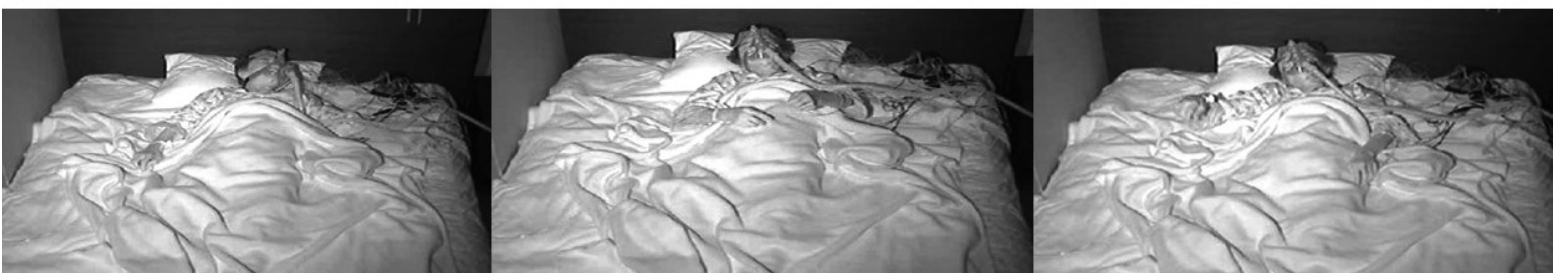

Figure 2. Initial hypnogram (A) has no REM sleep, but hypnogram during CPAP titration shows REM sleep (B). The events of sleep-disordered breathing, such as stridor and sleep hypopnea, are nearly eliminated. However, increased muscle tone on electromyogram channels (C) present as sleep talking and complex motor behaviors (D), which are compatible with REM sleep behavior disorder. (C) is a 120-s epoch polysomnogram during CPAP titration with $11 \mathrm{~cm} \mathrm{H} \mathrm{H}_{2} \mathrm{O}$ pressure during the stage of REM sleep (arrow). CPAP: continuous positive airway pressure, REM: rapid eye movement, Top 2 channels: electrooculogram, Chin 1-Chin 2: chin electromyogram, six channels consisting of F3-M2, F4-M1, C3-M2, C4-M1, O1-M2, O2-M1: electroencephalogram, LAT: electromyogram on left anterior tibialis muscle, RAT: electromyogram on right anterior tibialis muscle, Snore: snore sensor, Abd: abdomen respiratory effort channel, Chest: chest respiratory effort channel, Mflow: mask flow, PTAF: pressure transducer air-flow, EKG: electrocardiography, SaO2: oxyhemoglobin saturation by pulse oximetry, HR: heart rate.

pam, the first-line medication in the management of MSA patients with RBD, is known to worsen sleep apnea; ${ }^{10}$ this drug might exacerbate SDB, and consequently might influence sleep fragmentation in patients with MSA.

In the present report, we illustrated the case of a MSA patient with SDB who developed RBD during CPAP titration. To our knowledge, this is the first case of its kind, and hence merits reporting. Both RBD and SDB are common manifestations of MSA. ${ }^{1}$ Because RBD symptoms might affect CPAP adherence, physicians should carefully guide CPAP treatment in patients with MSA. Although MSA patients with SDB might have a history of RBD that has disappeared, clinicians should consider the presence of RBD symptoms during CPAP application with adequate pressure. Moreover, clonazepam treatment can exacerbate OSA and is associated with less optimal treatment outcomes in patients with $\mathrm{RBD} ;{ }^{11}$ hence, the choice of other classes of drugs without respiratory effects, such as melatonin, and more optimized CPAP treatment should be considered in MSA patients with SDB.

\section{Acknowledgments}

This research was supported by the 2017 scientific promotion program (Jeju National University).

\section{REFERENCES}

1. Gaig C, Iranzo A. Sleep-disordered breathing in neurodegenerative diseases. Curr Neurol Neurosci Rep 2012;12:205-217.

2. Ferini-Strambi L, Marelli S. Sleep dysfunction in multiple system atrophy. Curr Treat Options Neurol 2012;14:464-473.

3. Jang HJ, Kim B, Ryu HS, Lee GH, Lee SA. Two cases of REM sleep behavior disorder combined with severe obstructive sleep apnea: misdiagnosed as "pseudo-REM sleep behavior disorder" by diagnostic polysomnography. J Korean Sleep Res Soc 2013;10:62-65.

4. Patil SP, Schneider H, Schwartz AR, Smith PL. Adult obstructive sleep 
apnea: pathophysiology and diagnosis. Chest 2007;132:325-337.

5. Aldrich M, Eiser A, Lee M, Shipley JE. Effects of continuous positive airway pressure on phasic events of REM sleep in patients with obstructive sleep apnea. Sleep 1989;12:413-419.

6. Issa FG, Sullivan CE. The immediate effects of nasal continuous positive airway pressure treatment on sleep pattern in patients with obstructive sleep apnea syndrome. Electroencephalogr Clin Neurophysiol 1986;63:10-17.

7. Ghorayeb I, Yekhlef F, Bioulac B, Tison F. Continuous positive airway pressure for sleep-related breathing disorders in multiple system atrophy: long-term acceptance. Sleep Med 2005;6:359-362.

8. Ding Y, Hu YQ, Zhan SQ, Li CJ, Wang HX, Wang YP. Comparison study of polysomnographic features in multiple system atrophy-cerebellar types combined with and without rapid eye movement sleep behavior disorder. Chin Med J (Engl) 2016;129:2173-2177.

9. Vetrugno R, Alessandria M, D’Angelo R, et al. Status dissociatus evolving from REM sleep behaviour disorder in multiple system atrophy. Sleep Med 2009;10:247-252.

10. Zhang J, Li SX, Lam SP, Wing YK. REM sleep behavior disorder and obstructive sleep apnea: does one "evil" make the other less or more "evil"? Sleep Med 2017;37:216-217.

11. Li SX, Lam SP, Zhang J, et al. A prospective, naturalistic follow-up study of treatment outcomes with clonazepam in rapid eye movement sleep behavior disorder. Sleep Med 2016;21:114-120. 